\title{
Gold Magnetic Nanoparticles-based Chemiluminescent Immunoassay for Detection of Chloramphenicol in Milk
}

\author{
Linyu Wang ${ }^{1}$, Manwen Yao $^{2}$, Xiangyi Fang ${ }^{3}$, Peng Tang ${ }^{3}$ and Xi Yao ${ }^{1, a}$ \\ ${ }^{1}$ School of Electronic and Information Engineering, Xi'an Jiaotong University, Xi'an, PR China \\ 2 Tongji University, Shanghai, PR China \\ ${ }^{3}$ School of Science, Xi'an Jiaotong University, Xi'an, PR China
}

\begin{abstract}
CLIA) based on gold magnetic nanoparticles (Au-MNPs) were developed for rapid analysis of chloramphen icol (CAP) in milk sample. Anti-CAP antibodies were immobilized on the surfaces of Au-MNPs, luminol (Method I) and 2',6'-DiMethylcarbonylphenyl-10-sulfopropylacridinium-9-carboxylate 4'-NHS Ester, (NSP-DMAE-NHS, Method II) were exploited in competitive CLIA for CAP detection in milk using a homemade luminescent measurement system. The sensitivities and limits of detection (LODs) of the two methods were obtained according to the inhibition curves. It indicated that NSP-DMAE-NHS as luminescence reagent (reaction II) was more sensitive and effective than luminol (reaction I). The LOD of reaction II reached $0.008 \mathrm{ng} / \mathrm{ml}$ while it was $4 \mathrm{ng} / \mathrm{ml}$ in reaction I. Moreover, the linear range of the inhibition curve of the former was wider than that of the latter. Such results indicated that the proposed CLIA stratery employing NSP-DMAE-NHS was more sensitive than other immunoassay method for CAP detection.
\end{abstract}

\section{Introduction}

Chloramphenicol (CAP), a kind of cheap and effective bacteriostatic broad-spectrum antibiotics, was widely used in animal husbandry to treat kinds of sensitive bacteria infection. However, it was found that CAP had serious adverse effects against human bodies, leading to bone marrow depression and even aplastic anemia [1-2]. Due to these side effects, it was banned from use in animal feeds and food in the European Union (EU), Switzerland, USA and other countries [3-4].

Various methods have been developed for detection of CAP residues in animal food. Conventional methods like microbiological methods [5] were not sensitive for CAP and were totally obsolete. Gas/liquid chromatography (GC/LC) [6-7] and gas/liquid chromatography-mass spectrometry (GC-MS/LC-MS) [8-10] are internationally recognized but expensive, complicated to operate, and not suitable for detecting lots of samples. Immunoassays including radio immunoassay (RIA) [11], enzyme-linked immunosorbent assay (ELISA) [12-13] have been reported frequently to screen CAP. RIAs have high sensitivity but exists radioactive waste, and ELISAs have shortcoming of higher limit of detection (LOD). Chemiluminescent immunoassays (CLIAs) have some features such as non-radioactive wastes, simple instrumentation, the very low limit of detection (LOD), high sensitivity, wide dynamic range and rapid analysis time in only several seconds. Those characteristics make it broadly used in clinical, environment, food and factory analysis in recent years [14-15].

\footnotetext{
${ }^{\mathrm{a}}$ Corresponding author : xyao@mail.xjtu.edu.cn
} 
Magnetic nanoparticles had been widely used as solid carry in RIA, ELISA and CLIA, due to: (i) the easier manipulation because the magnetic nanoparticles can be controlled in magnetic field instead of centrifuging repeatedly, (ii) the increased surface area as well as (iii) the faster assay kinetics because the nanoparticles are in suspension [16]. In addition, due to the improved washing and separation steps, the matrix effect is minimized in complex samples [17]. Gold magnetic nanoparticles (Au-MNPs) have outstanding characteristics with combining the superparamagnetism of magnetic nanoparticles and the ability of being modified by biomolecules on gold surface.

Chemiluminescence reactions which exploited luminol (or N-(4-Aminobutyl)-N-ethylisoluminol, ABEI) and an enhancer (such as p-iodophenol) by hydrogen peroxide $\left(\mathrm{H}_{2} \mathrm{O}_{2}\right)$ in the presence of horseradish peroxidase (HRP) were reported widely [18]. Weeks [19] synthesized acridinium esters (AE)-NHS [4-(2-Succinimidyfoxycarbonylethyl) phenyl-10methylacridinium -9-carboxylate fluorosulfonate] and it has high luminous efficiency with no need of catalyst and enhancer. However, the thermal stability of AE-NHS is not so good that many researches have been done to syntheses more stable acridinium esters (2',6'-DiMethylcarbonylphenyl-10-sulfopropylacridinium-9-carboxylate 4'-NHS Ester). Which is an ideal chemical luminescence immunoassay marker for the thermal stability and hydrolytic stability. This paper explored the Au-MNPs-based CLIA methods for CAP detection in milk samples respectively with luminol (reaction I) and NSP-DMAE-NHS (reaction II) as luminescence reagent.

\section{Materials and methods}

\subsection{Synthesis of $\mathrm{Fe}_{3} \mathrm{O}_{4} @ \mathrm{SiO}_{2} @ A u$ magnetite nanoparticles}

$\mathrm{Fe}_{3} \mathrm{O}_{4}$ Magnetic nanoparticles (Fe-MNPs) were prepared by solvothermal synthesis reaction with some modifications. Typically, $\mathrm{FeCl}_{3} 6 \mathrm{H}_{2} \mathrm{O}(1.35 \mathrm{~g})$ was first dissolved in $40 \mathrm{~mL}$ ethylene glycol to form a clear solution, followed by addition of polyethylene glycol (1.0 g), afterward, sodium acetate $(3.6 \mathrm{~g})$ was added with stirring. The mixture sealed in autoclave. It was heated to $200^{\circ} \mathrm{C}$ for $10 \mathrm{~h}$. The black products were washed and dried in a vacuum oven at $80^{\circ} \mathrm{C}$.

Fe-MNPs $(340 \mathrm{mg}$ ) were dispersed in solvent that contained $250 \mathrm{~mL}$ ethanol and $30 \mathrm{~mL}$ deionized water under ultrasonic vibration for five minutes. Then $3 \mathrm{~mL}$ ammonia (28 wt.\%) and $2 \mathrm{~mL}$ TEOS were added slowly with strring and the reaction was continuous stirred for another 15 hours. We separated the $\mathrm{Fe}_{3} \mathrm{O}_{4} @ \mathrm{SiO}_{2}$ with a magnet. Then, we used APTES as a amination reagent. $\mathrm{Fe}_{3} \mathrm{O}_{4} @$ $\mathrm{SiO}_{2}(150 \mathrm{mg})$ in suspension under ultrasonic vibration in $50 \mathrm{ml}$ ethanol were added $100 \mathrm{uL}$ APTES, which reacted 48 hours at room temperature. The solid products were collected, washed with deionized water and ethanol and re-dispersed in $100 \mathrm{ml}$ water.

Gold colloids with the size of $30 \mathrm{~nm}$ were prepared by reduction of $\mathrm{HAuCl}_{4}$ with sodium citrate. Then, $30 \mathrm{~mL}$ of the amino-functionalized $\mathrm{Fe}_{3} \mathrm{O}_{4} @ \mathrm{SiO}_{2}$ nanoparticles dispersion was added dropwise to $100 \mathrm{ml}$ of the citrate-stabilized gold colloids. With stirring for 1 hour, the resulting product was separated magnetically and washed with water.

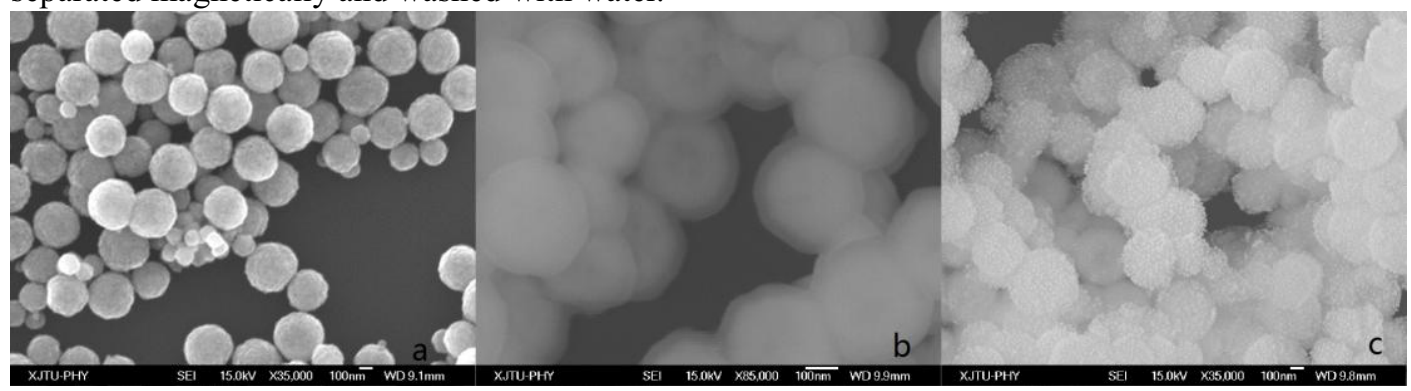

Figure.1. a SEM of $\mathrm{Fe}_{3} \mathrm{O}_{4} \quad$ b SEM of $\mathrm{Fe}_{3} \mathrm{O}_{4} @ \mathrm{SiO}_{2} \quad$ c SEM of $\mathrm{Fe}_{3} \mathrm{O}_{4} @ \mathrm{SiO}_{2} @ \mathrm{Au}$ 


\subsection{Anti-CAP antibody immobilization on Au-MNPs}

The immobilization process of the anti-CAP antibodies on the Au-MNPs is shown in Fig 3(A). Carboxyl-(Au-MNPs) which was formed by immersing Au-MNPs in a solution of 11-Mercaptoundecanoic acid (MUA, $5 \mathrm{mM}$ ) in ethanol for $16 \mathrm{~h}$ at room temperature was washed twice with MES (2-(N-Morpholino) ethanesulfonic acid) buffer (25 mM, pH5.12). Afterwards, the carboxyl-(Au-MNPs) was re-suspended in MES buffer and activated by incubation with a 1:1 ratio mixture of $200 \mathrm{mM}$ EDC (N-ethyl-N'-(3-dimethylaminopropyl) carbodiimide hydrochloride ) and 50 mM NHS (N-hydroxysulfosuccin-imide) in MES buffer for $30 \mathrm{~min}$. After washed three times with MES buffer and distilled water respectively, $100 \mu \mathrm{l}$ of the Au-MNPs were incubated with $100 \mu 1$ of anti-CAP antibody at $37^{\circ} \mathrm{C}$ for 2 hours. After incubation, the supernatant was removed and washed three times with PBS $(0.01 \mathrm{M}, \mathrm{pH} 7.4)$ in a magnetic field.

\subsection{Determination of the tracers' optimized amount}

Before construction of standard curves for different concentration of CAP in two chemiluminescence reactions, the optimized amount of tracers (CAP-HRP and CAP-(rabbit IgG)-(NSP-DMAE-NHS) conjugates) could be determined by binding them on the antibody-immobilized Au-MNPs with different concentrations. Fig.2 (A) shows the emission intensity (CPS) of the chemiluminescence corresponding to different amount of CAP-HRP. The concentration of CAP-HRP was $0.6 \mathrm{mg} / \mathrm{ml}$. It can be seen that the chemiluminescence intensity increased with the increase of CAP-HRP amount in the range of 50-200 $\mu 1$ and the CPS reached saturation at $200 \mu 1$. This extraordinary result may be explained by steric effect. In this case, CAP-HRP saturation amount was $200 \mu \mathrm{l}$. In consideration of ensuring higher test sensitivity in the competitive reaction and tracer consumption, the optimal CAP-HRP amount of $180 \mu 1$ was selected as the fixed volume for competitive reaction.
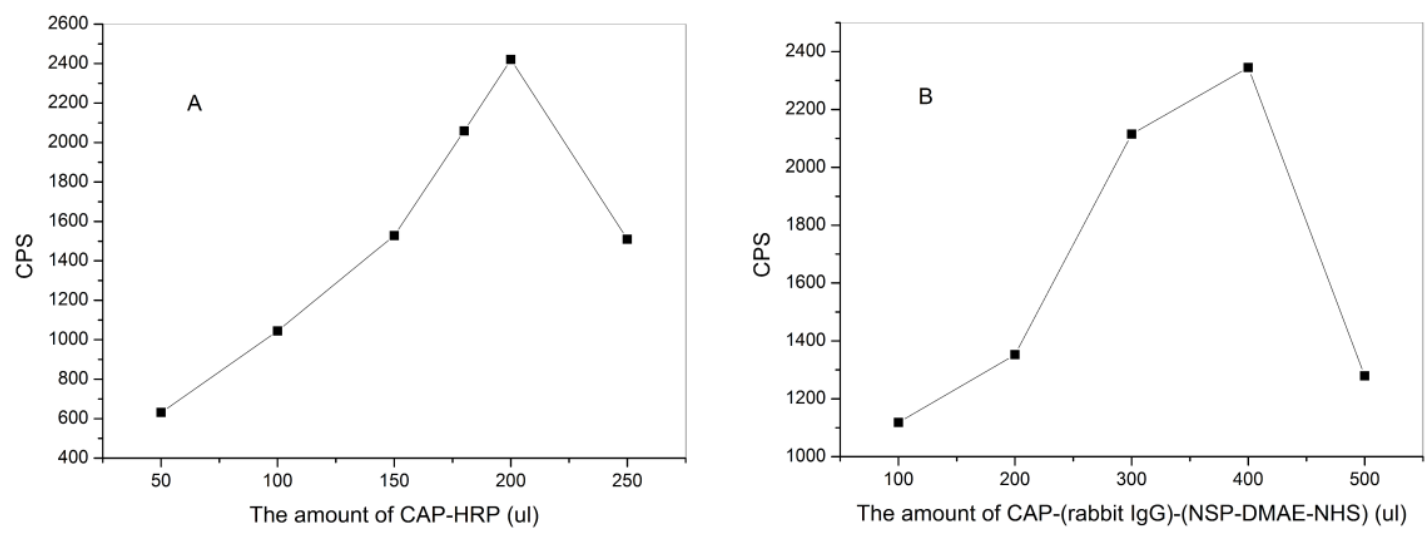

Figure.2. (A) The emission intensity versus the amount of CAP-HRP (B) The emission intensity versus the amount of CAP-(rabbit IgG)-(NSP-DMAE-NHS)

Fig.2 (B) shows the CPS according to different amount of CAP-(rabbit IgG)-(NSP-DMAE-NHS). There also existed the steric effect and the saturation amount of this tracer was $400 \mu 1$. In consideration of the same reason, the optimal amount of CAP-(rabbit IgG)-(NSP-DMAE-NHS) of 300 $\mu \mathrm{l}$ was determined for competitive reaction in target CAP measurement.

\subsection{Establishment of CLIA method based on Au-MNPs for CAP detection}


The process of reaction I is shown in Fig.3 (B). CAP succinate sodium and commercial milk powder were dissolved in deionized water to prepare the standard milk samples in which the concentration of the milk powder was $1 \mathrm{mg} / \mathrm{ml}$ and the concentrations of the CAP succinate sodium were $0.01,0.1,1$, 10, 100 and $1000 \mathrm{ng} / \mathrm{ml}$ respectively. The immobilized anti-CAP antibodies on Au-MNPs, which were prepared by the procedures described in the previous section, were allowed to react with a mixture of certain amount of CAP-HRP tracer and the spiked milk samples. The mixture was allowed to incubate at $37{ }^{\circ} \mathrm{C}$ for 2 hours with gentle shaking. Then the Au-MNPs were washed with PBS in magnetic field. In this study, the substrate solution was made of luminol $(0.5 \mathrm{mM})$, p-iodophenol $(0.7 \mathrm{mM})$ and $\mathrm{H}_{2} \mathrm{O}_{2}$ $(14 \mathrm{mM})$ in Tris-HCl buffer $(20 \mathrm{mM}, \mathrm{pH} 8.6)$ according to a former experiment. A volume of $100 \mu 1$ of the mixture of luminol and p-iodophenol (1:1) was added to the Au-MNPs, followed by the injection of $100 \mu \mathrm{l}$ of $\mathrm{H}_{2} \mathrm{O}_{2}$ by a peristaltic pump to induce chemiluminescence of the antibody-bound CAP-HRP on the Au-MNPs. At the same time, the chemiluminescence emission was measured by the luminescent measurement system.

The process of reaction II is shown in Fig.3 (C). The preparation of spiked milk samples was the same as reaction I except that the concentrations of the CAP succinate sodium were $0.0001,0.001$, $0.01,0.1,1,10 \mathrm{ng} / \mathrm{ml}$ respectively. CAP-(rabbit IgG)-(NSP-DMAE-NHS) conjugates immobilized on $\mathrm{Au}-\mathrm{MNPs}$. Then the particles were washed by PBS in a magnetic field. Here, $\mathrm{HNO}_{3}+\mathrm{H}_{2} \mathrm{O}_{2}$ and $\mathrm{NaOH}$ acted as the pre-trigger and trigger respectively to induce chemiluminescence emission. A volume of $100 \mu 1$ of the pre-trigger was previously added to the Au-MNPs and $100 \mu$ lof trigger was injected into the tube by a peristaltic pump to induce chemiluminescence of the antibody-bound conjugates of CAP-(rabbit IgG)-(NSP-DMAE-NHS) on Au-MNPs. At the same time, the chemiluminescence emission intensity was measured versus time by the luminescent measurement system.
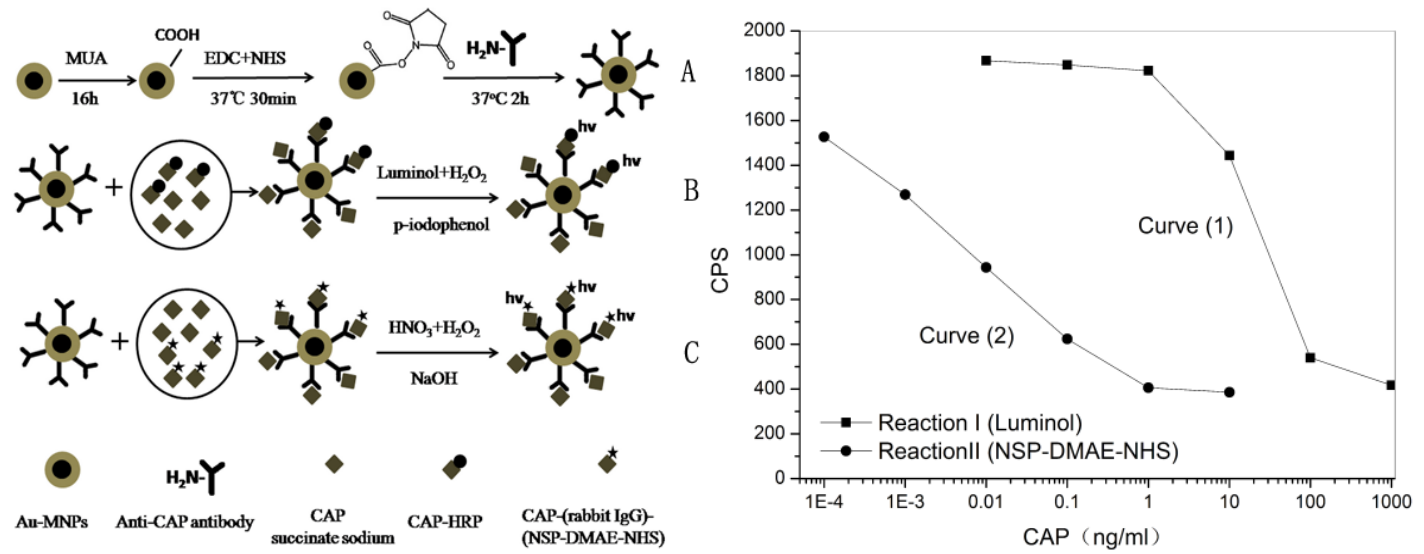

Figure.3. two CLIA reactions for CAP detection.

Figure.4. Calibration curves for detection of CAP in milk

\section{Conclusions}

In Fig.4, curve (1) showed the relationship of chemiluminescence intensity (CPS) versus the logarithm scale of concentrations of CAP succinate sodium in the range of $10^{-2}-10^{3} \mathrm{ng} / \mathrm{ml}$. The curve (2) showed the CPS versus the logarithm scale of concentrations of CAP succinate sodium in the range of $10^{-4}-10$ $\mathrm{ng} / \mathrm{ml}$. The $\mathrm{IC}_{50}$ values (the competitor concentration that causes $50 \%$ growth inhibition) of reaction I and reaction II were about $30 \mathrm{ng} / \mathrm{ml}$ and $0.03 \mathrm{ng} / \mathrm{ml}$. The LOD value of reaction I and reaction II were approximately as $4 \mathrm{ng} / \mathrm{ml}$ and $0.008 \mathrm{ng} / \mathrm{ml}$. In view of $\mathrm{IC}_{50}$, the sensitivity of reaction II was improved 1000 -folds compared with that of reaction I. This results indicates that applying NSP-DMAE-NHS as the chemiluminescent reagent is more sensitive and has a much wider measurement range than luminol in the CLIA. These results demonstrated that Reaction II was much more sensitive for the 
detection of CAP than some previously reported methods such as ELISA $\left(\mathrm{IC}_{50}\right.$ of $10.5 \mathrm{ng} / \mathrm{ml}$ and LOD of $0.2 \mathrm{ng} / \mathrm{ml},[20]$ ), the pretreatment-free immunochromatographic assay (LOD of $10 \mathrm{ng} / \mathrm{ml}$, [21]) and the time-resolved fluoroimmunoassay (TRFIA) (LOD of $0.05 \mathrm{ng} / \mathrm{g}$, [22]).

In conclusion, Reaction II which used NSP-DMAE-NHS-labeled CAP-rabbit $\operatorname{IgG}$ as a tracer performed higher sensitivity and wider linear measurement range than reaction I which employed luminol with a tracer of CAP-HRP. Based on this, We will carry on to apply our system and method to detect other residues in food, and to make this biosensor commercialized in the future.

\section{Acknowledgement}

This work was supported by the National Natural Science Foundation of China [Grant No. 81371642]; the 111 Project (B14040).

\section{References}

1. M. J. Kasten, Mayo. Clin. Proc. 74, 825 (1999)

2. X. Z. Shi, A. B. Wu, S. L. Zheng, R. X. Li, D. B. Zhang, J. Chromatogra. B. 850, 24 (2007).

3. I. S. Park, N. Kim, Clin. Chim. Acta. 578, 19 (2006)

4. A.Y. Kolosova, J.V. Samsonova, A. M. Egorov, Food. Agr. Immunol. 12, 115 (2000)

5. J. D. Louvois, J. Antimicro. Chemoth. 9, 253 (1982)

6. V. Cerkvenik-Fajs,. Biomed. Chromatogr. 20, 985 (2006)

7. H. Y. Shen, H. L. Jiang, Anal. Chim. Acta, 535, 33 (2005)

8. M. X. Xie, Y.Liu, Y. M. Qiu, J. Han, Y. Z. Liu, Chinese. J. Anal. Chem. 33, 1 (2005)

9. K. Fujita, H. Ito, M. Nakamura, M. Watai, M. Taniguchi, J-AOAC 91, 1103 (2008)

10. S. B.Turnipseed, J. E. Roybal, A. P. Pfenning, P. J. Kijak, Anal. Chim. Acta. 483, 373 (2003)

11. D. Arnold, A. Somogyi, J-AOAC 68, 984 (1985)

12. V.Gaudin, N.Cadieu, P. Maris, Food. Agr. Immunol. 15, 143 (2003)

13. N.Liu, S.Q. Song, L. Lu, D.X. Nie, Z. Han, X.L. Yang, Food. Agr. Immunol. 25, 523 (2014)

14. S. Lin, S.Q. Han, Y.B. Liu, W.G. Xu, Anal. Bioanal. Chem. 382, 1250 (2005)

15. X.Q. Tao, W. J. Wang, Z. H. Wang, X. Y. Cao, Luminescence 29, 301 (2014)

16. T. Laube, S. V. Kergaravat, S. N. Fabiano, S. R. Hernandez, Biosens. Bioelectron. 27, 46 (2011)

17. E. Zacco, M. I. Pividori, S. Alegret, Anal. Chem. 78, 1780 (2006)

18. C. L. Xu, C. F. Peng, K. Hao, Z.Y. Jin, W. K. Wang, Luminescence 21,126 (2006)

19. I. Weeks, I. Beheshti, F. McCapra, A. K. Campbell, Clin. Chem. 29, 1474 (1983)

20. N. Sai, Y. P. Chen, N. Liu, G. G. Yu, P. Su, Y. Feng, Z. J. Zhou, Talanta 82, 1113 (2010)

21. N. A. Byzova, E. A. Zvereva, A. V. Zherdev, S. A. Eremin, Talanta 81, 843 (2010)

22. J. Z. Shen, Z. Zhang, Y. Yao, W. M. Shi, Y.B. Liu, Anal. Chim. Acta. 575, 262 (2006) 